\title{
Predicting Changes in Workflow Resulting from Healthcare Information Systems: Ensuring the Safety of Healthcare
}

\author{
$\overline{\text { Andre Kushniruk, Elizabeth Borycki, Shige Kuwata and Joseph Kannry }}$
}

\begin{abstract}
This paper describes an approach to studying medical error and workflow that can be applied to help ensure the safety of new healthcare information systems. The approach focuses on identification of usability problems resulting from implementation of new information technology, as well as identification of problems related to changes in workflow. The paper illustrates how the approach can be applied in the simulation-based analyses of emerging healthcare information systems. The paper includes discussion of the application of an approach to identifying inadvertent changes in healthcare workflow that may result from design issues in a range of information technologies including medication order entry systems. General implications for the design, development and evaluation of safer healthcare information systems are discussed. It is argued that there is a need for thorough simulation-based testing of systems under a variety of conditions before they are released in order to ensure the safety of healthcare.
\end{abstract}

\section{Introduction}

The need to reduce medical error has become a driving force and motivation for the widespread deployment of healthcare information systems. Indeed, a number of highly influential publications have supported the notion that introduction of information technology, such as computerized patient records, will lead to decreased medical error (Bates et al. 1998). However, recent work by the authors (Kushniruk et al. 2004; Kushniruk et al. 2005) and others (Koppel et al. 2005) has indicated that poorly designed user interfaces and systems may actually increase the likelihood of occurrence of certain types of errors. On the basis of such work, the necessity of ensuring that new information systems not only reduce medical error but also that they do not inadvertently add new types of errors (or inadvertently change healthcare workflow) is becoming recognized as being a critical issue in healthcare. This paper extends the authors' previous work in developing novel methodologies based on usability testing and simulations for the identification and prediction of technology-induced errors prior to release of new healthcare information systems. In addition, in this paper we will demonstrate how the approach can be used to identify how implementation of healthcare information systems may inadvertently affect workflow, and how this can be detected prior to release of the system. The paper describes a recent case study in order to illustrate how the approach can be used to accurately pinpoint potential issues and changes in workflow that may arise in the implementation of a new medication order entry system. 


\section{Application of Simulations in Detecting and Predicting Technology-Induced Error}

In life-critical industries such as aviation and nuclear power, a range of simulation approaches have been used in order to deliver effective and safe information systems, with an extremely low tolerance for error introduced by technology. This has included use of computer-based simulations to model complex physical and mechanical interactions in the design and testing of applications. Such simulations can be considered to be completely "in the box," since they use a computer program that simulates real-life activities. A second category of simulations includes more realistic testing of software applications under conditions that approximate real-life conditions. Such simulations incorporate both physical and cognitive stimuli that are representative of real-life situations "outside the box" in order to test how software will behave under different conditions. In this paper we describe how simulations of realistic work activities, inspired by methods used in domains such as the aviation industry, can be applied in studying health information systems.

The overall approach to testing health information systems extends a method used in medical education, known as the "standardized patient" (Kushniruk et al. 1996). In this case a physician or other healthcare professional interacts with an information system (e.g., enters patient data into a computerized patient record system) while interacting with someone playing the role of a patient (who may be answering questions posed by the healthcare professional on the basis of a "patient script"). This approach builds on related work in usability testing, where representative users of a system (e.g., physicians or nurses) are observed while they carry out representative tasks (e.g., entering a medication) using a system under study (Kushniruk and Patel 2004).

An example of this approach is work by Kushniruk and colleagues (Kushniruk et al. 1996) using videorecordings of doctors interacting with an information system while they carried out a data retrieval task (e.g., obtaining data about patients from the system). Subjects in such studies may be asked to "think aloud" while carrying out the task (which is audiorecorded), or in the case of interaction with a simulated patient they may be asked to carry out a doctor-patient interview while using the system under study (with the dialogue between the doctor and patient audiorecorded, while the computer screens are videorecorded). A number of evaluations have been conducted that have been able to identify problems with health information systems and user interfaces under relatively realistic conditions prior to their release. For example, work based on simulation testing by Kushniruk et al. (2005) identified a range of usability problems with the interface to a commonly used handheld personal digital assistant (PDA) application for prescription writing.

\section{Methodological Approach}

Our methodological approach has emerged from recent advances in usability engineering and the study of human-computer interaction in patient safety. This research uses low-cost software for recording computer screens of a system under study in conjunction with low-cost videorecording equipment and computer systems used by a physician or healthcare worker (see Kushniruk and Patel 2004 for details of recording methods used). Our early work in the study of healthcare applications allowed us to isolate the cognitive effects of technology (e.g., interface design and content) and their potential for inadvertently inducing or facilitating medical errors. In the case study described in this paper, the focus is on extending this approach to analyzing the impact of a new information technology upon both cognitive and physical interactions (i.e., clinician workflow) in a realistic clinical setting, including consideration of both the computer system and the work environment.

\section{Case Study: Evaluating the Impact of a Medication Order Entry System}

A simulation approach was used to study a new medication order entry system to be deployed in a teaching hospital. The system was designed to allow users (i.e., doctors and nurses) to
Figure 1.

A nurse subject interacting with a medication order entry system while being videorecorded.

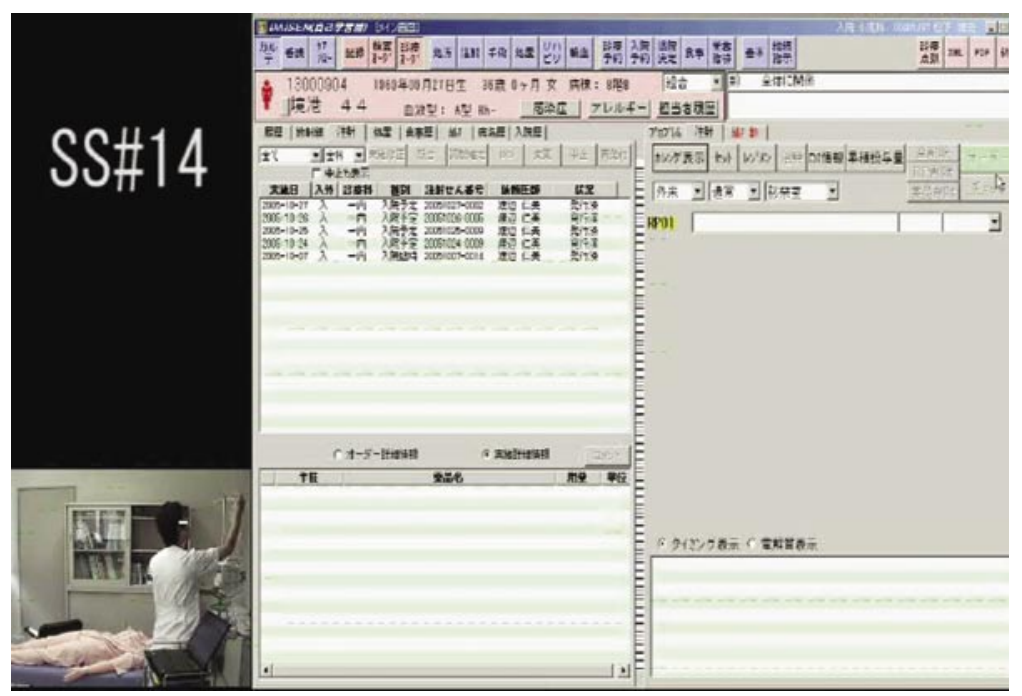


obtain information about what medications to give patients and to record the administration of the medication in a computer system. This system is similar to many systems currently being deployed in hospitals around the world. The computer component of the medication order entry system was also integrated with bar-coding technology that allows the doctor or nurse to scan the wristband of the patient to identify the patient and to also scan labels on medication bags. Figure 1 illustrates the setup, with a nurse working with a medication order entry system while being videorecorded (physical activities are recorded using a camcorder on a tripod; in addition, all computer screens are automatically recorded using Hypercam ${ }^{\odot}$ screen recording software).

Sixteen subjects, consisting of doctors and nurses, were given written instructions for entering medications for a list of simulated patients. The subjects interacted with both the computer system (via a keyboard as shown in Figure 1), to obtain instructions for administering medication, and the "patient," actually a mannequin with a bar-coded wristband. In the simulation, subjects were specifically instructed to interact with the computer system and the dummy patient (e.g., to hang intravenous medication bags) just as in a real situation. In order to record the use of the system in the simulation, we employed a digital video camera on a tripod to record the interactions of the subject with both the computer system and the patient.

Subjects were asked to use the computer application to enter the patient's name, obtain the list of medications to give the patient, administer the medication (to the dummy patient) and then record the administration in the computer application. All computer screens were recorded, while subjects' interactions with a dummy patient were recorded using the camcorder. At the end of the session the subjects were also interviewed (and the interviews audiorecorded).

In order to analyze the data collected, first the audio portion of the recorded sessions was transcribed in its entirety (including the interviews at the end of each session - see Figure 2 for the transcripts from one nurse subject), and then annotated by the experimenters by reviewing the videorecordings of the computer screens and subjects' physical activities (e.g., actually hanging medication bags). In Figure 2 the numbers on the left-hand side refer to the video counter corresponding to the actual actions of the subject. The latter portion of Figure 2 also contains the transcript of the interview with the subject (a nurse) conducted immediately following completion of the simulation task.

From analysis of the recordings of the subjects carrying out the study tasks, it was observed that the system imposed a very sequential order of activities in order to document the medication administration. Also, from the interview portion, ergonomic issues related to difficulty in subjects actually scanning the information on labels on medication bags were noted by subjects. In addition, a number of computer-related issues were brought up
Figure 2. Annotated transcript of subject (nurse) administering medication, and post-task interview

\section{Medication order information obtained by nurse}

00:14 Nurse searches for patient on the computer

00:45 Nurse views order list on the screen

00:51 Nurse selects medication order from list

00:55 Verification screen appears

Nurse walks over to patient to check identification

00:59 Nurse talks to patient - "Nice to meet you. I will now give you an IV drip."

01:09 Nurse scans patient identification (from patient's wristband)

01:10 Verification screen automatically updates

Nurse walks back to computer

01:25 Nurse views execution information on the computer

\section{Nurse walks over to patient and sets medication bag}

Nurse walks back to computer

03:15 Nurse confirms administration of medication on the computer

Post-task Interview:

Experimenter: Did you find any difficulty with the task?

Subject: I'm used to this operation, but sometimes it is hard to use the bar-code reader when the bar code is not clearly printed.

Experimenter: What difficulties did you have with the bar-code reader?

Subject: There are no problems when we have both a printed order and a label on the bottle (we can use either of them, because there are the same bar codes on both). But if the bar code is only on the bottle with its rough surface, I have often pushed its surface to flatten it, and scan it many times until I can read the bar code correctly.

Experimenter: Do you find any difficulty during the workflow process?

Subject: Sometimes I could not open the record of the patient whom I was giving a medication to, because another nurse or doctor was opening the record at the same time.

by subjects in such interviews, such as the inability to record medication administration when the patient's record is "locked out" by other users of the system who are accessing the system at the same time as the nurse or doctor is attempting to administer medication.

In addition to identifying potential sources of specific problems that would arise from implementation of the new system, from the analysis of all the subjects it was observed that introduction of the computer actually led to a major change in the process of medication administration. This was characterized by a serialization of the workflow process that could not be deviated from - for example, as shown in the annotated transcript in Figure 2, the physician or nurse would have to administer one 
medication at a time, first accessing the computer, moving to the patient, scanning the identification band on the patient's wrist, moving back to the computer for details, then back to the patient to administer that drug and finally back to the computer to record the administration prior to administering the next medication (which is repeated each time for each medication). Thus, compared to the previous workflow, the new system imposed a rigid order of activities for medication entry.

\section{Results: Impact of the System on Healthcare Workflow}

Analysis of the data collected indicated that use of the system would have the impact of changing the workflow of the physicians and nurses considerably, compared to the manual paperbased procedures they used before the system was introduced. For example, in hanging intravenous (IV) medications, prior to introduction of the system physicians and nurses could hang the drugs in a parallel manner (e.g., checking the paper medication administration record, verifying and arranging all the medication bags and equipment, checking the client's identification band, explaining the medications to the client, hanging and administering the medications and documenting them) (Kozier et al. 2002). In cases in which only one or two medications needed to be administered, it appeared that the system was not problematic. However, when we adjusted our simulations to include medication lists with many medications, the serial process of administering medications became not only tedious for users but under time constraints led to perceived stress by users. Under normal conditions, this might lead to increased safety in medication entry by providing a structured and standardized procedure for medication entry; however, from our simulations it was also clear that under certain conditions (e.g., when there is a need to administer a number of medications under time pressure) the new system might also result in cognitive overload, necessitating complete bypass of the system by users under emergency or stressful situations. It should be noted that such consequences were not anticipated by the designers of the system.

\section{Lessons Learned}

Using the approach described above, we are examining the impact of a range of healthcare information systems, including decision support tools, Web-based health-related information resources and computerized patient record systems. In these studies we have worked with system designers and implementers (using simulation approaches) to identify and rectify usability problems. On the basis of our current work described here, we have also found that a close relationship exists between specific usability problems and the potential for occurrence of specific types of medical error. Furthermore, introduction of a new healthcare information system may result in unexpected changes in workflow that may also be related to medical error under certain circumstances. It is therefore essential that methods such as those based on simulation testing be developed and refined for assessing the potential impact of a range of healthcare systems prior to their release in real clinical environments.

As illustrated in the case study, in order to provide accurate and predictive information about the impact of healthcare information systems, simulation studies need to consider not only the interaction of the user of the system with the computer, but also the complex interplay between use of the computer application, the patient and other technologies involved in the workflow. This can be observed in the real-world environment (e.g., a hospital room) by employing portable, low-cost videoand audiorecording equipment. It is essential that we observe interactions of typical users with new information technologies in realistic settings prior to release of these systems, particularly as healthcare workers may be unaware of the impact of systems on workflow. Understanding how the system may impact the interaction of the healthcare worker with the patients, coworkers and other individuals in the actual clinical environments where systems will be implemented is key. In the case of complex systems, such as medication order entry systems, this also includes consideration of other technologies (e.g., barcoding scanning devices, IV pumps, etc.) that may be integrated into the new process and not just the computer system in isolation. This includes consideration of the physical placement of different components (e.g., computer, patient bed, bar-code scanner, etc.) and how they integrate into the overall process of healthcare.

Predicting potential impact of systems requires simulations that represent a wide range of cases or scenarios. This includes not only routine, non-urgent conditions, but also urgent or time-constrained ones. For example, one of the main findings in our case study was that use of the system serialized the process of order entry, which under certain conditions might result in increased cognitive load. Thus, under some conditions advantages can be outweighed by disadvantages. Such feedback leads to corrective action early in the implementation process, and has an impact on cost savings as well as increasing the likelihood of user acceptance of systems. More studies similar to that described here are needed to see emerging information technologies in healthcare, including use of portable tablet devices, mobile handheld devices, mobile carts and workstations at the bedside.

\section{Discussion/Conclusions}

Many types of simulations have been employed in life-critical industries to assess the potential for new systems to inadvertently induce error. In this paper we have described an approach to analyze and detect potential technology-induced error and to identify inadvertent changes to workflow that may impact 
safety. We are currently working on refining the methodological approach and creating an empirically based classification of problems encountered by subjects based on the results of our ongoing simulation studies. Usability-based approaches involving simulations of clinical contexts allow for considerable power in predicting potential problems with systems prior to their release. This approach to in-depth study of the interaction of healthcare workers with information systems and devices prior to their release in healthcare settings will be essential to ensure that the systems we introduce are safe, and that we reap the full benefits of technological innovation.

\section{About the Authors \\ Andre Kushniruk, PhD, is Associate Professor and Director, School of Health Information Science, University of Victoria.}

Elizabeth Borycki is Assistant Professor, School of Health Information, and Adjunct Assistant Professor, School of Nursing, University of Victoria.

Shige Kuwata is Assistant Professor, Division of Medical Informatics, Tottori University Hospital, Tottori, Japan.

Joseph Kannry is Chief, Division of Clinical Informatics, Mount Sinai Medical Center, New York, New York.

Please direct correspondence to: Andre Kushniruk, PhD, Associate Professor and Director, School of Health Information Science, University of Victoria, Human \& Social Development Building A202, 3800 Finnerty Road (Ring Road), Victoria, BC V8P 5C2. Tel: (250) 721-8578. Fax: (250) 472-4751. E-mail: andrek@uvic.ca.

\section{References}

Bates, D.W., L.L. Leape, D.J. Cullen, N. Laird, L.A. Petersen, J.M. Teich, E. Burdick, M. Hickey, S. Kleefield, B. Shea, M. Vander Vliet and D.L. Seger. 1998. "Effect of Computerized Physician Order Entry and a Team Intervention on Prevention of Serious Medication Errors." Journal of the American Medical Association 280(15): 1311-16.

Borycki, E. and A.W. Kushniruk. 2005. "Identifying and Preventing Technology-Induced Error Using Simulations: Application of Usability Engineering Techniques." Healthcare Quarterly 8: 99-105.

Koppel, R., J. Metlay, B. Cohen, R. Abaluck, S. Localio, S. Kimmel and B. Strom. 2005. "Role of Computerized Physician Order Entry Systems in Facilitating Medication Errors." Journal of the American Medical Association 293: 1197-203.

Kozier, B., G. Erb, A. Berman and S. Snyder. 2002. Techniques in Clinical Nursing: Basic to Intermediate Skills. Upper Saddle River, NJ: Prentice Hall.

Kushniruk, A.W., D.R. Kaufman, V.L. Patel, Y. Levesque and P. Lottin. 1996. "Assessment of a Computerized Patient Record System: A Cognitive Approach to Evaluating an Emerging Medical Technology." M.D. Computing 13(5): 406-15.

Kushniruk, A.W. and V.L. Patel. 2004. "Cognitive and Usability Engineering Approaches to the Evaluation of Clinical Information Systems." Journal of Biomedical Informatics 37: 56-57.

Kushniruk, A.W., M. Triola, E. Borycki, B. Stein and J. Kannry. 2005. "Technology Induced Error and Usability: The Relationship between Usability Problems and Prescription Errors When Using a Handheld Application." International Journal of Medical Informatics 74: 519-26.

Kushniruk, A., M. Triola, B. Stein, E. Borycki and J. Kannry. 2004. "The Relationship of Usability to Medical Error: An Evaluation of Errors Associated with Usability Problems in Use of a Handheld Application for Prescribing Medications." MedInfo - World Congress on Medical Informatics 2004: 1073-76.

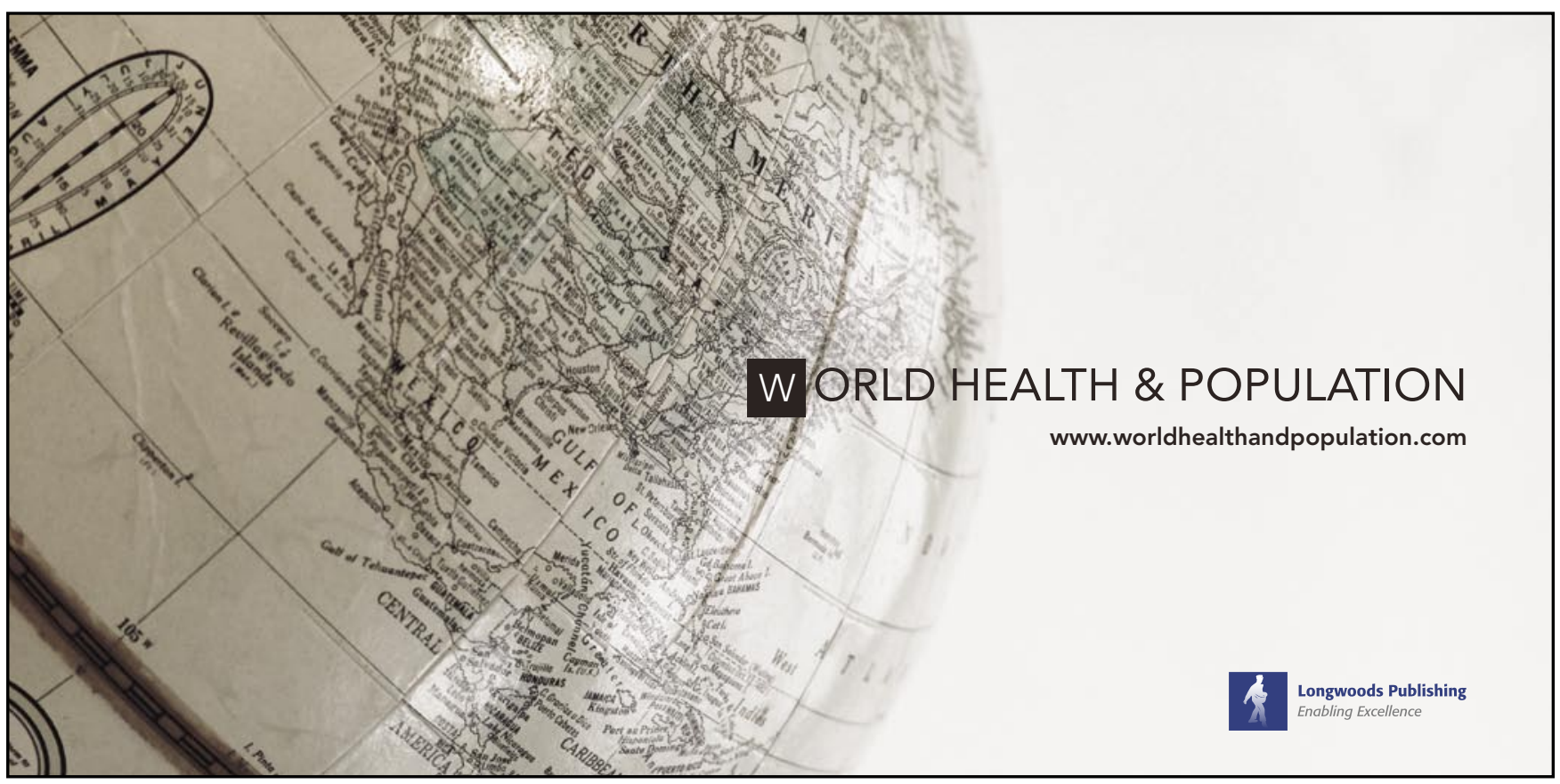

